\title{
Fault Diagnosis Method for Hydraulic Pump Based on Fuzzy Entropy of Wavelet Packet and LLTSA
}

\author{
https://doi.org/10.3991/ijoe.v14i02.7845 \\ Wang Fei $\left.{ }^{\bowtie}\right)$, Fang Liqing, Qi Ziyuan \\ Mechanical Engineering College, Shijiazhuang, China \\ m15536259885@163.com
}

\begin{abstract}
As the vibration signal characteristics of hydraulic pump present non-stationary and the fault features is difficult to extract, a new feature extraction method was proposed.This approach combines wavelet packet analysis techniques, fuzzy entropy and LLTSA (liner local tangent space alignment) which is one of typical manifold learning methods to extracting fault feature. Firstly, the vibration signals were decomposed into eight signals in different scales, then the fuzzy entropies of signals were calculated to constitute eight dimensions feature vector. Secondly, LLTSA method was applied to compress the high-dimension features into low-dimension features which have a better classification performance. Finally, the SVM (support vector machine) was employed to distinguish different fault features. Experiment results of hydraulic pump feature extraction show that the proposed method can exactly classify different fault type of hydraulic pump and this method has a significant advantage compared with other feature extraction means mentioned in this paper.
\end{abstract}

Keywords - wavelet packet analysis; fuzzy entropy; LLTSA; feature extraction; hydraulic pump; fault diagnosis

\section{$1 \quad$ Introduction}

In fault diagnosis of hydraulic pump, the vibration signal contains abundant running status information, analyzing the vibration signal is the most commonly used method in fault diagnosis process [1]. Vibration signal which was obtained from the hydraulic pump presents complicated, non-linear and non-stationary, so how to accurately extract the fault feature from these signals effectively is the key to improve the accuracy rate of fault diagnosis.

Wavelet transform is a kind of signal time scale analysis method in multiresolution, and it has the ability to characterize the local features of the signals in time and frequency domain[2]. But this method has some problems, it only decomposes the low frequency signals rather than the high ones and its resolution ratio decreases when increase the frequency. Wavelet packet analysis is a method which could solve this problem, it is a more elaborate method than wavelet decomposition and performed better in high-frequency information subdivision. Wavelet packet analysis can decompose both the low frequency signals and the high ones, and also adaptively 
select corresponding band with signal spectrum according to signal features $[3,4]$. The wavelet packet theory has the good time-frequency characteristic and it can be availably used for the noise suppression processing of the digital signal, thus wavelet packet has a wider range of applications in contrast with wavelet transform. Hydraulic pump system is difficult to analyze, the wavelet packet analysis method can be used to achieve different scale fault information from the original vibration signal.

In the late $1940 \mathrm{~s}$, Shannon introduced the concept of entropy into the field of information theory applied to the uncertainty of the event, after that the entropy gradually generalized. In recent years, the information entropy, the sample entropy, the approximate entropy and the permutation entropy have been widely used in mechanical fault diagnosis, using all kinds of entropies as reflect characteristic parameters of fault information and had achieved favorable results[5,6,7]. The concept of entropy is transferred to the fuzzy set theory, and then fuzzy entropy can be obtained. The fuzzy entropy is a index to measure the complexity and irregular degree of time series, and it is an improved type of the sample entropy or approximate entropy. As there are different types of hydraulic pump faults, the results of fault vibration signal with wavelet packet decomposition will be changed under different scales of structure characteristics, the complexity and irregularity degree, and its fuzzy entropy value can produce significant change, so the different scales of fuzzy entropy can be used as a feature vector for feature extraction $[8,9,10]$.

The fault feature which extracted preliminarily always contains redundant information, so it is necessary to use the dimension reduction algorithm to reduce the vector dimensions of fault feature in order to improve the accuracy of fault identification[11]. Linear local tangent space alignment (LLTSA) is a new kind of popular learning algorithm, it has a better dimension reduction effect compared with the principal component analysis (PCA) algorithm, and it can fully extract the low dimensional characteristic from the high-dimensional nonlinear data and has a very good nonlinear complex information processing capabilities [12,13].

Based on the above analysis, this paper put forward the hydraulic pump fault diagnosis methods based on wavelet packet decomposition, fuzzy entropy and LLSTA. Multi-scale analysis ability of wavelet packet decomposition was used to obtain different scales of hydraulic pump fault information, the different scales of fuzzy entropy were extracted as the characteristics of the hydraulic pump under different fault states, and then LLTSA was used to conduct feature dimension reduction which could get fault features with low dimension, high sensitivity and good clustering performance. At last, the working state and fault type of hydraulic pump were distinguished by support vector machine. The effectiveness and superiority of this proposed method have been proved through fault signal diagnosis experiments of hydraulic pump and comparison with other methods. 


\section{Methods}

\subsection{Wavelet Packet Analysis}

Wavelet packet analysis is a more accurate decomposition method than the wavelet decomposition, the former can improve the accuracy in high-frequency decomposition and increase the time-frequency resolution compared with the latter. Therefore, wavelet packet decomposition has stronger signal analysis ability. In the practical application, decomposition and reconstruction of wavelet packet decomposition is realized by combining wavelet packet coefficient and high-low frequency filter convolution.

The following process is the wavelet packet analysis method:

Step1: wavelet packet decomposition

$$
\begin{aligned}
& d_{i, j, 2 m}=\sum_{k} h(k-2 i) d_{k, j+1, m} \\
& d_{i, j, 2 m+1}=\sum_{k} g(k-2 i) d_{k, j+1, m}
\end{aligned}
$$

Step2: wavelet packet reconstruction

$$
d_{i, j+1, m}=\sum_{k} h(i-2 k) d_{k, j, 2 m}+\sum_{k} g(i-2 k) d_{k, j, 2 m+1}
$$

Among them, $d_{i, j, m}$ is the jth wavelet packet coefficients of the mth layer of a node. $\mathrm{h}(\mathrm{k})$ and $\mathrm{g}(\mathrm{k})$ are coefficients of expansion.

In the process of feature extracting for a signal by using wavelet packet analysis, the choosing of wavelet kernel function and decomposition levels can greatly affect the extracting performance. Daubechies wavelet kernel function is orthogonal and its time domain waveform is exactly similar to the hydraulic pump vibration waveform, on the other hand, Daubechies function can exactly reflect the mutation point in pump vibration signal because of its suitable tight support interval, so the Daubechies wavelet kernel function can describe the vibration signal very well. Based on the above considerations, the Daubechies4 function was chose as the wavelet kernel function. the choosing of decomposition levels should reflect the vibration signal energy distribution changes and have a low level influence on the calculation speed, considering the sampling frequency and signal characteristics, in this paper, the decomposition level is determined to be 3 layers.

Fig.1 is three-level wavelet packet decomposition. $\mathrm{S}$ is the initial signal. $\mathrm{L}$ is low frequency components of signals. $\mathrm{H}$ is high frequency components of signals. The serial number is the layer number of wavelet decomposition, which is also called scale parameters. 


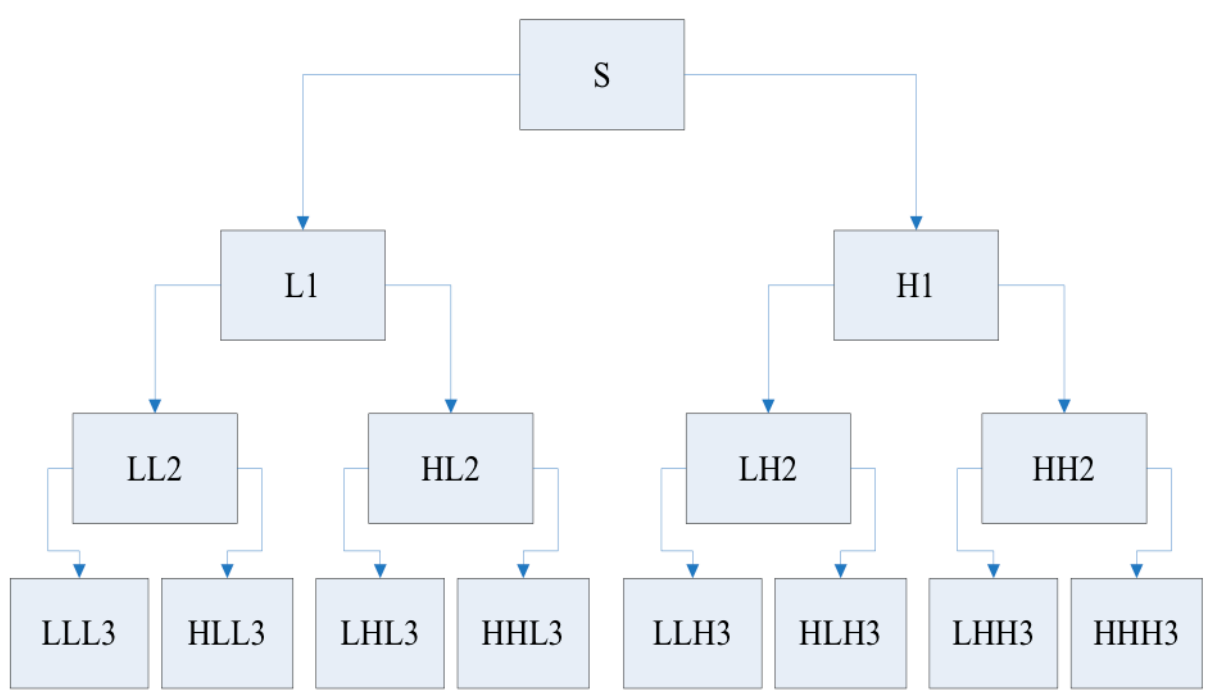

Fig. 1. 3 layer wavelet packet decomposition structure diagram

\subsection{Fuzzy Entropy}

Similar to sample entropy and approximate entropy[14,15], fuzzy entropy is a method to measure the time series complexity by numerical, on the other hand, it can describe the probability of generating the new model when pattern dimension changed. The more numerical value, the more complex. Compared with sample entropy, fuzzy entropy introduced the fuzzy membership function to replace the hard threshold sample entropy criterion in sample entropy, contrast with approximate entropy, fuzzy entropy used the fuzzy membership function to blur the vector similarity measurement, so fuzzy entropy changed continuously and smoothly when the parameter changed, it have a low degree of dependency and sensitivity to parameter variation and a more stable statistical results[16]..

The following description is the calculation process of fuzzy entropy;

Step 1:For an $N$-point time series, the m-dimensional vector $\{u(i): 1 \leq i \leq N\}$ according to the order was built.

$$
X_{i}^{m}=\left\{u(i), u(i+1), \cdots, u(i+m-1)-u_{0}(i)\right\}, i=1,2, \cdots, N-m+1
$$

In the above expression, $X_{i}^{m}$ means $m$ continuous value of $\mathrm{u}$ which has been taken out mean value $u_{0}(i)$ from the $i$ point.

$$
u_{0}(i)=\frac{1}{m} \sum_{j=0}^{m-1} u(i+j)
$$


Step 2: $d\left[X_{i}^{m}, X_{j}^{m}\right]$ was defined as the distance of $X_{i}^{m}$ and $X_{j}^{m}$, it represent the maximum absolute difference value between the element $X_{i}^{m}$ of and $X_{j}^{m}$ :

$$
\begin{gathered}
\max _{k \in(0, m-1)}\left\{\left|\left(u(i+k)-u_{0}(i)\right)-\left(u(j+k)-u_{0}(j)\right)\right|\right\} \\
i, j=1,2, \mathrm{~L}, N-m, i \neq j
\end{gathered}
$$

Step 3: The similarity $D_{i j}^{m}$ of vector $X_{i}^{m}$ and the vector $X_{j}^{m}$ was defined according to the fuzzy function $\mu\left(d_{i j}^{m}, n, r\right)$ :

$$
D_{i j}^{m}=\mu\left(d_{i j}^{m}, n, r\right)=e^{-\left(d_{i j}^{m} / r\right)^{n}}
$$

The fuzzy function $\mu\left(d_{i j}^{m}, n, r\right)$ is exponential function, $n$ represents the gradient of fuzzy function boundaries and $r$ means the width.

Step 4: Defined average similarity function as follows:

$$
\phi^{m}(n, r)=\frac{1}{N-m} \sum_{i=1}^{N-m}\left(\frac{1}{N-m-1} \sum_{j=1, j \neq i}^{N-m} D_{i j}^{m}\right)
$$

Step 5: Similarly, for the dimension $m+1$, repeat the step 1 to step 4 and get the function as follows:

$$
\phi^{m+1}(n, r)=\frac{1}{N-m} \sum_{i=1}^{N-m}\left(\frac{1}{N-m-1} \sum_{j=1, j \neq i}^{N-m} D_{i j}^{m+1}\right)
$$

Step 6: Fuzzy entropy is defined as:

$$
\text { FuzzyEn }(m, n, r)=\lim _{N \rightarrow \infty}\left[\ln \phi^{m}(n, r)-\ln \phi^{m+1}(n, r)\right]
$$

When $\mathrm{N}$ is a finite number, the formula can be expressed as:

$$
\operatorname{FuzzyEn}(m, n, r, N)=\ln \phi^{m}(n, r)-\ln \phi^{m+1}(n, r)
$$

In the calculation process of fuzzy entropy, there are four parameters which should be considered: embedding dimension $m$, similarity tolerance $r$, ambiguity function gradient $n$, data length $N$. According to the findings in document 17, when $m=1$ or 2 , $r=0.1 S t d-0.25 S t d$ (Std is the standard deviation of time-series data $\boldsymbol{u}(\boldsymbol{i})$ ), the obtained sample entropies have reasonable statistical properties; According to the recommendations from references 18 , gradient $n$ generally use the smaller integer, such as 2 or 3 ; the analysis results of reference 19 showed that when the data length is more than 500 , the value of entropy will tend to be stable. From the analysis above, the parameters to calculate fuzzy entropy were set as $m=2, r=0.2 \mathrm{Std}, n=2, N=1024$. 


\subsection{Liner Local Tangent Space Alignment (LLTSA)}

Data dimension reduction is a method which takes high-dimensional data to project onto a low dimensional space by a linear or non-linear mapping and get its lowdimensional representation. Local tangent space alignment (LTSA) is a typical nonlinear manifold learning algorithm; the core idea is to use a certain point and its neighboring tangent space to represent the local geometric properties of these data points, the low-dimensional coordinates will be obtained from the projection on local tangent space. Then these local low dimensional coordinates will be taken to construct global low dimensional coordinates by permutation transformation. However, due to the high requirement to the data (data must be denseness and should satisfy uniform distribution and local linear) and is sensitive to the curvature and noise of the data, LTSA is limited in practical application. As the local maximal linear scaling of LTSA, LLTSA takes into full account to local information and the topological structure of the high dimensional dataset, it combined LTSA with adaptive linear block, contrasted difference value between the euclidean distance and geodesic distance with threshold $\theta$ to construct the local block data. LLTSA could strictly control local linear features of nonlinear data by geometric perturbation and have low loss of information after dimension-reduced processing compared with LTSA dimension reduction results.

Basically, LLTSA has the following several steps:

Step 1: Principal component analysis (PCA) projection. Using PCA to map the data to the subjective subspace. $\boldsymbol{A}_{\mathrm{PCA}}$ represents transformation matrix of PCA, at the same time in order to convenient expression, $\boldsymbol{X}$ is used to represent PCA subspace data set.

Step 2: Determine the neighborhood. Search the neighboring data points $x_{i}$ by KNN (K-Nearest Neighbor) method, namely, construct distance matrix of all data points by Euclidean distance, then find $k$ same approximation points $x_{j}$ of $x_{i}$ by analyzing the distance matrix.

Step 3: Acquire the local information. The $d$ feature vectors of $d$ maximum characteristic value of $X_{i} H_{k}\left(X_{i}=\left[x_{i 1}, \mathrm{~L}, x_{i k}\right]\right)$ are calculated and $H_{k}=I-e e^{T} / k$. The matrix $V_{i}$ are constituted by $d$ feature vecetors.

Step 4: Construct permutation matrix. Construct matrix through local accumulative:

$$
\begin{aligned}
& \boldsymbol{B}\left(\boldsymbol{I}_{i}, \boldsymbol{I}_{i}\right) \leftarrow \boldsymbol{B}\left(\boldsymbol{I}_{i}, \boldsymbol{I}_{i}\right)+\boldsymbol{W}_{i} \boldsymbol{W}_{i}^{T}(i=1, \mathrm{~L}, N) \\
& \text { Initialize } \boldsymbol{B}=0, \boldsymbol{I}_{i}=\left\{i_{i}, \mathrm{~L}, i_{k}\right\} \text { is } \mathrm{k} \quad \text { index neighbor points of } x_{i}, \\
& \boldsymbol{W}_{i}=\boldsymbol{H}_{k}\left(i-\boldsymbol{V}_{i} \boldsymbol{V}_{i}^{\mathrm{T}}\right)(i=1, \mathrm{~L}, N) .
\end{aligned}
$$

Step 5: Calculate mapping. Calculate the eigenvalues of the generalized characteristics and characteristic vector: 


$$
\begin{gathered}
\boldsymbol{X} \boldsymbol{H}_{N} \boldsymbol{B} \boldsymbol{H}_{N} \boldsymbol{X}^{\mathrm{T}} \alpha=\lambda \boldsymbol{X} \boldsymbol{H}_{N} \boldsymbol{X}^{\mathrm{T}} \alpha \\
\lambda_{1}, \lambda_{2}, \mathrm{~L}, \lambda_{n}\left(\lambda_{1}<\lambda_{2} \mathrm{~L}<\lambda_{n}\right) \text { is } \alpha_{1}, \alpha_{2}, \mathrm{~L}, \alpha_{d}, \quad \boldsymbol{A}_{\mathrm{LLTSA}}=\left(\alpha_{1}, \alpha_{2}, \mathrm{~L}, \alpha_{d}\right) \text {. So the } \\
\text { transfer matrix is } \boldsymbol{A}=\boldsymbol{A}_{\mathrm{PCA}} \times \boldsymbol{A}_{\mathrm{LLTSA}}, \boldsymbol{X} \rightarrow \boldsymbol{Y}=\boldsymbol{A}^{\mathrm{T}} \boldsymbol{X}_{\mathrm{ORG}} \boldsymbol{H}_{N}
\end{gathered}
$$

\subsection{The model of fault diagnosis based on fuzzy entropy of wavelet packet and LLTSA}

Because the original machinery vibration signal is relatively complicated, it is difficult to accurately extracting the fault feature directly. Using the multi-resolution characteristic of wavelet packet analysis, the wavelet packet decomposition was combined with fuzzy entropy to extract the fault signal features under multi-scale to compose fault feature vector, the high-dimensional fault feature vector was reduced into low dimensional vector by using LLSTA method. In this way, fault features were enhanced and the data complex rate was reduced. Through this method the accuracy of fault diagnosis was expected to be improved, the specific steps are as follows:

Step 1: Wavelet packet decomposition and reconstruction: Using Daubechies4 wavelet packet to decompose the original signal into 3 layer and got 8 decomposing sequences, reconstructed the 8 sequences of different frequency bands and each signal contained different information .

Step 2: Calculation of fuzzy entropy: using the formula (10) to calculate the 8 wavelet packet reconstruction signal and the fuzzy entropy separately, and then the eight-dimensional fuzzy entropy feature vector were constituted as:

$$
\boldsymbol{T}=[\mathrm{e} 1, \mathrm{e} 2, \mathrm{e} 3, \mathrm{e} 4, \mathrm{e} 5, \mathrm{e} 6, \mathrm{e} 7, \mathrm{e} 8]^{\mathrm{T}}
$$

Step 3: Feature dimension reduction: LLSTA method was used to 8 wavelet fuzzy entropy feature dimension reduction, low dimensional popular feature vector.

Step 4: Classification: computing to low dimensional feature input of SVM classification recognition.

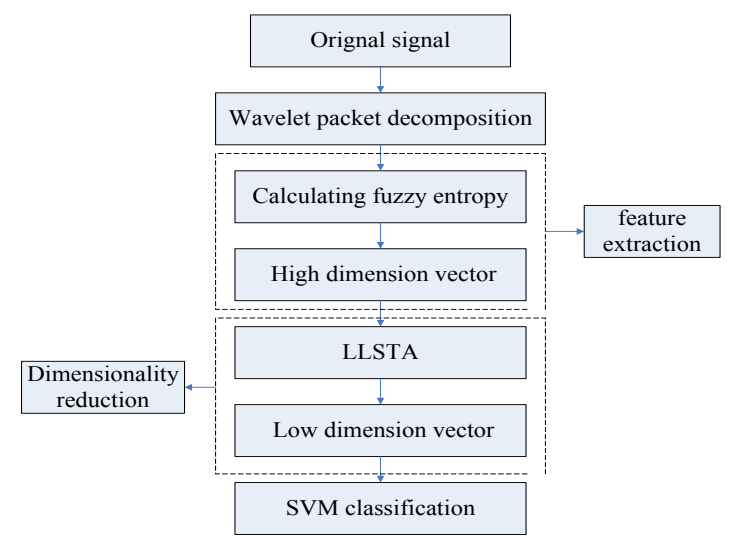

Fig. 2. Fault diagnosis model 


\section{$3 \quad$ Fault Test analysis}

\subsection{Data Acquisition}

In this paper, the experiment was conducted on the hydraulic pump experiment platform. Fig. 3 is the hydraulic pump experiment platform. The hydraulic pump test platform consists of drive system, pressure regulating system, control system, signal monitoring, acquisition and display system and cooling system. In driving system, the power of the drive motor is $90 \mathrm{KW}$, in the converter under the mediation, the speed in the range from 0 to $3000 \mathrm{r} / \mathrm{min}$ under the control of the frequency converter, so it can adapt to different tests of hydraulic pumps. The pressure regulating system ensures that the test platform can withstand pressure up to $40 \mathrm{MPa}$.

The hydraulic pump in the experiment is a cam plate type axial piston pump, the model type was SY-10MCY14-1EL, the number of plunger was 7, the rated speed was $1500 \mathrm{rpm}$, the main relief valve pressure was $10 \mathrm{Mpa}$.The vibration signal were acquired by the piezoelectricity accelerating speed sensor installed on the end cover of the hydraulic pump, the type of the sensor is $603 \mathrm{C} 01$. The sampling frequency was $20 \mathrm{kHz}$, the vibration signal was stored by DAQ-9171 measure system made by NI company. Hydraulic pump of the experiment is shown in fig. 4. In this paper, four kinds of fault states were set: Normal working state $(\mathrm{N})$, single plunger loose slipper (1P), double plungers loose slipper (2P), piston shoes wear (S). The two types of faults were shown in fig. 5. Fig.6 is the time domain waveform of four kinds of fault states, which shows that the failure vibration signals are complex non-stationary modulation signal and reflect regular impact properties, it can not directly distinguish the these fault status from the vibration signal. Therefore, the signal need for further processing.

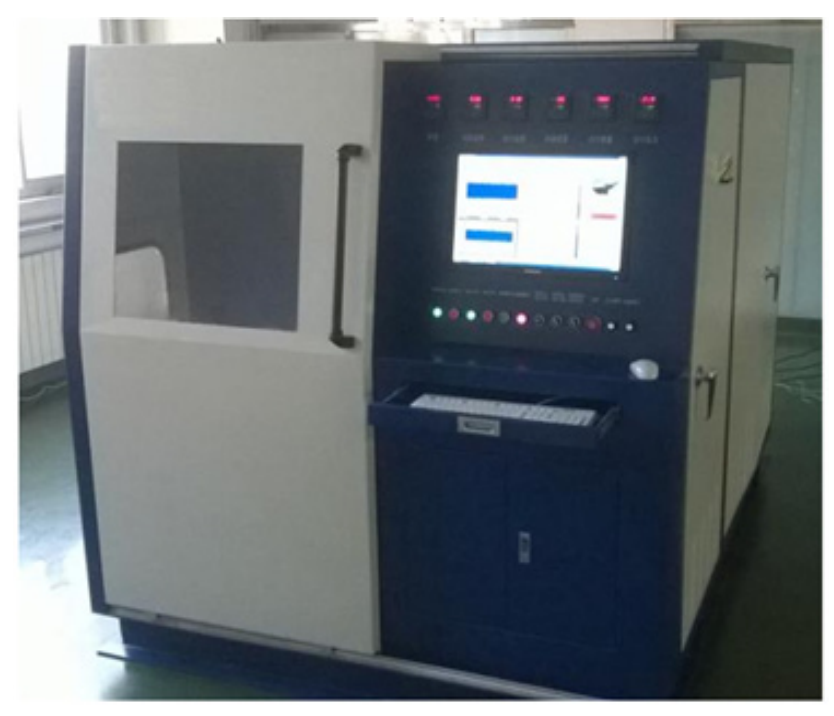

Fig. 3. The experiment platform of hydraulic pump 
Paper-Fault Diagnosis Method for Hydraulic Pump Based on Fuzzy Entropy of Wavelet Packet and ...

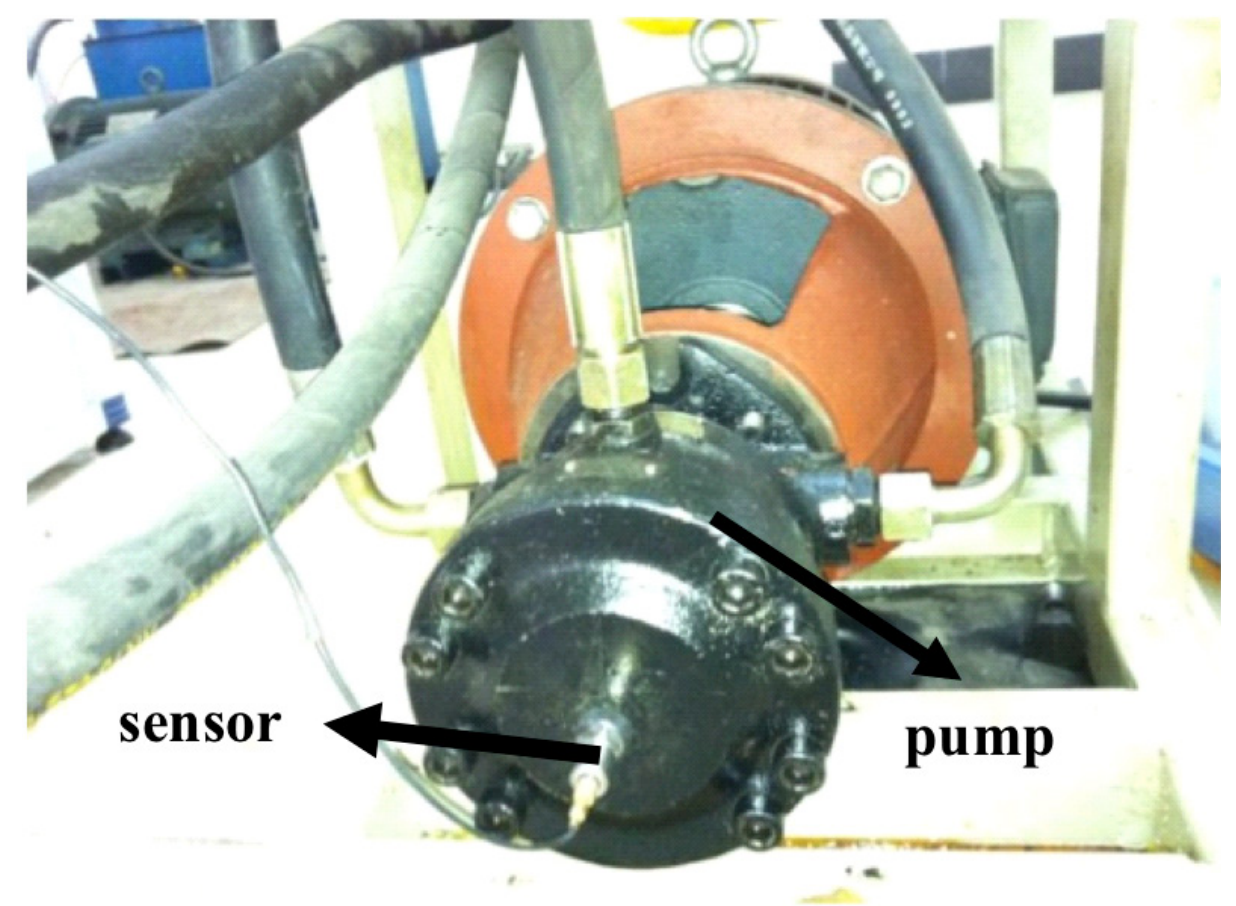

Fig. 4. Test device in hydraulic pump experiment

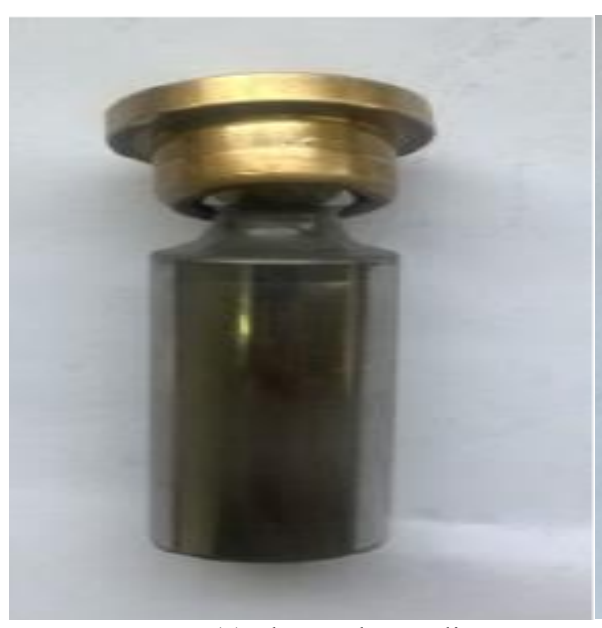

(a) plunger loose slipper

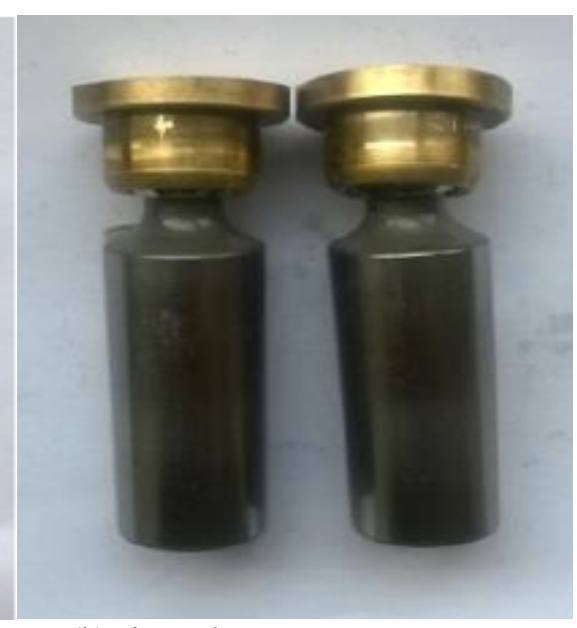

(b) piston shoes wear

Fig. 5. The fault types of the hydraulic pump 

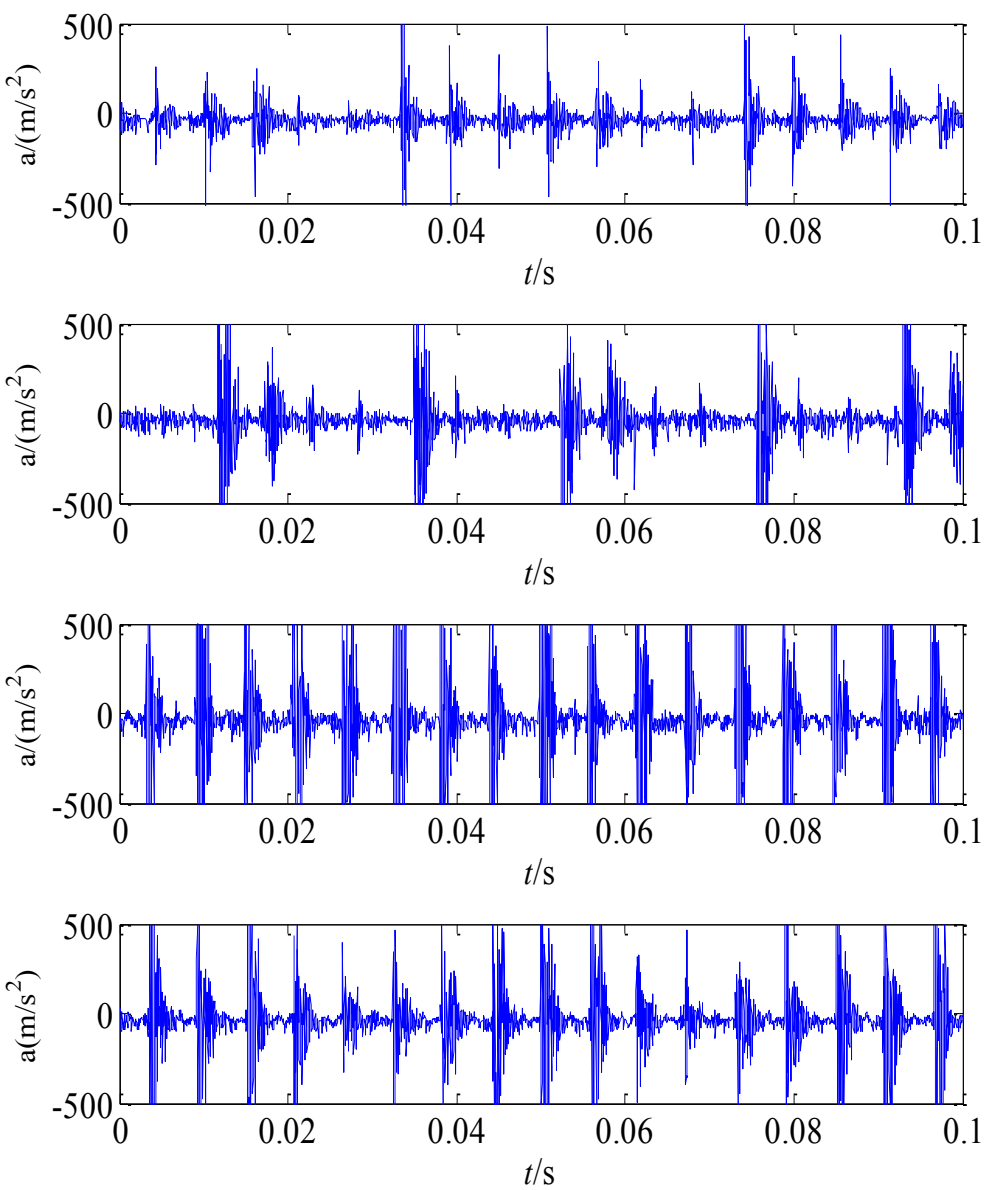

Fig. 6. The original time domain signal $(\mathrm{N}, 1 \mathrm{P}, 2 \mathrm{P}, \mathrm{S})$

\subsection{Fault Feature Extraction}

40 groups of samples were selected from the time domain signals of 4 kinds of fault states, and the data length of each group was 1024. Calculating the fuzzy entropy (Fuzzy-en) of four kinds of original signal and each average value of the fuzzy entropy was got. As comparison, the 4 average values of sample entropy (Sample-en) were calculated. The values of the two kinds of entropy are shown as follows:

Table 1. the fuzzy entropy and sample entropy of original signal

\begin{tabular}{|l|c|c|c|c|}
\hline \multicolumn{1}{|c|}{ fault } & N & 1P & 2P & S \\
\hline fuzzy entropy & 0.927 & 0.7624 & 0.3616 & 0.7265 \\
\hline Sample entropy & 1.0431 & 0.74 & 0.4964 & 0.7426 \\
\hline
\end{tabular}


Under normal condition, the hydraulic pump is running smoothly and the vibration signal is distributed evenly, the uncertainty degree is relative high, the fuzzy entropy value of normal condition $(\mathrm{N})$ is greater than the other three kinds of fault signal $(1 \mathrm{P}, 2 \mathrm{P}, \mathrm{S})$ entropy. The fault degree of double loose slipper is more serious, the impulse signal has a strong regularity and the uncertainty of the signal distribution decreases, thus the fuzzy entropy is minimum. The fuzzy entropy of single loose slipper and the sliding shoe wear is similar and it is difficult to identify the two fault condition. The average sample entropy of the signals follows the same rules. Using fuzzy entropy is easier to distinguish the singer plunger loose and sliding shoe wear than using sample entropy, the statistical result of fuzzy entropy is more stability and fuzzy entropy has certain advantages in descripting the fault features in compare with sample entropy.

In order to effectively extract the fault characteristics of each fault condition and improve the fault recognition rate, DB4 wavelet was used to carry out 3 layer wavelet packet decomposition for each group of sample signal, then the decomposition coefficients of the 8 frequency band obtained from decompose were reconstructed. Each signal contained the different frequency band vibration information of original signal. Fig. 7 is the results of the 3 layer wavelet packet decomposition of the single plunger loose fault. Fuzzy entropies were calculated for each sample signal of 8 wavelet packet reconstruction signal and 8 dimensional feature vectors were established.

Fig. 8 (a) is the of the average value of the fuzzy entropy of the wavelet packet in four states. In order to reflect the advantages of fuzzy entropy, the sample entropy and approximate entropy of each reconstructed signal were calculated by the same method. Its distribution diagram is shown in fig. (b), (c).
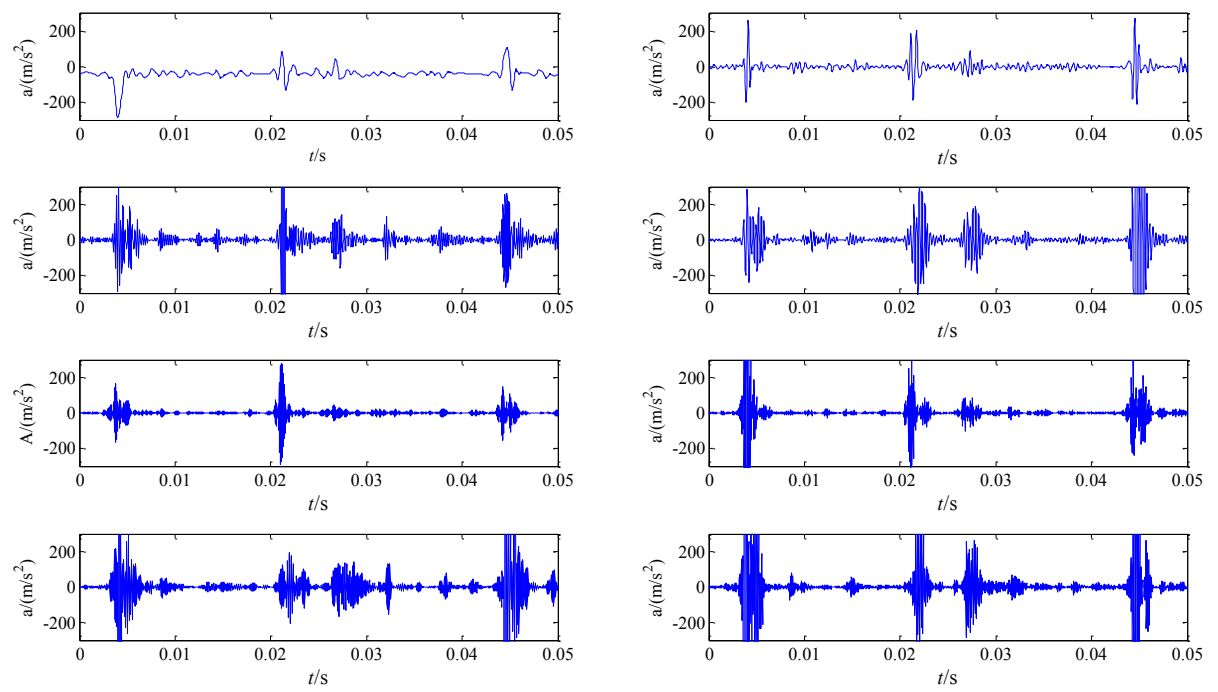

Fig. 7. Wavelet packet reconstitution signals of loose slipper fault 


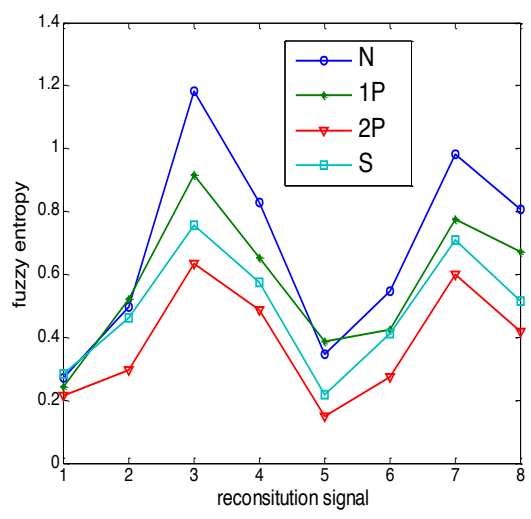

(a)

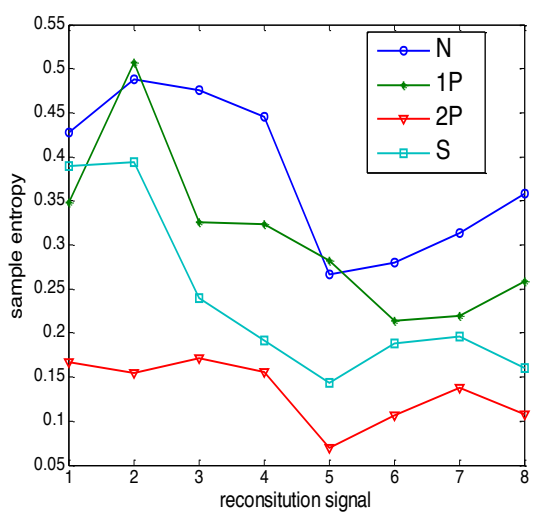

(b)

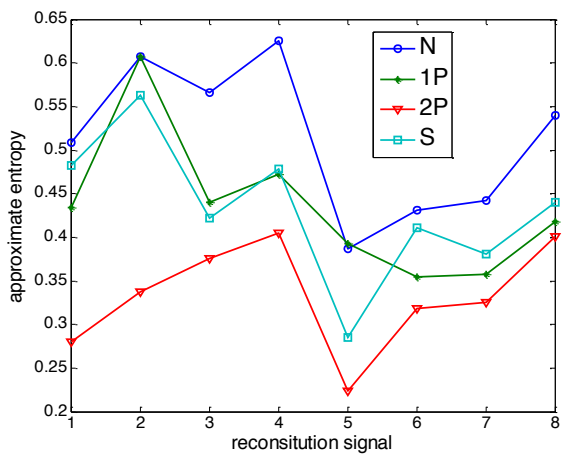

(c)

Fig. 8. The distribution of average wavelet packet entropy. (a)Fuzzy entropy (b) Sample entropy. (c)Approximate entropy

Comparing the above three kinds of wavelet packet entropy. From the diagram (a), the 4 kinds of fault condition can well be distinguished by using the fuzzy entropy. The distribution of fuzzy entropy has a strong regularity and the distribution trend is consistent, especially the 4 performance of signal 3, 4, 6 and 7. From the fig. (b), it can be seen that the sample entropy of normal working state $(\mathrm{N})$ and single plunger loose slipper (1P) have some confusion in 2 and 5 reconstructed signals, and distribution regularities of the four state sample entropies are not strong; Fig. (c) shows the confusion of approximate entropy between single and double plunger loose slipper (1P and $2 \mathrm{P}$ ) is more serious, it is difficult to distinguish the two types of fault.

Through the analysis, it is further indicated that compared with the wavelet packet sample entropy and approximate entropy, the fuzzy entropy of wavelet packet is more stable and the clustering performance is better, the fuzzy entropy has advantage to distinguish the different fault states. 


\subsection{Feature Dimension Reduction}

Constituted by eight reconstructed signal fuzzy entropy value which was from the decomposed wavelet packet, eight-dimensional fault feature vector was reduced to three-dimensional one as the target by using the LLTSA method and parameter $\mathrm{k}$ equals twelve. For comparison, the LTSA and PCA methods were both used for dimension of feature vector. Fig. 6 is about the rendering of state classification after feature dimension reduction by PCA, LTSA and LLTSA (each group of the four kinds has 20 samples). It is evident that LLTSA has better effect of low-dimensional feature extraction and cluster compared with PCA and LTSA from fig. 9.

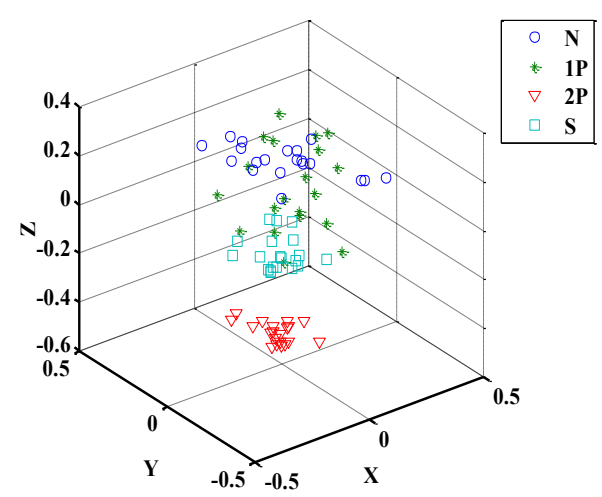

(a)

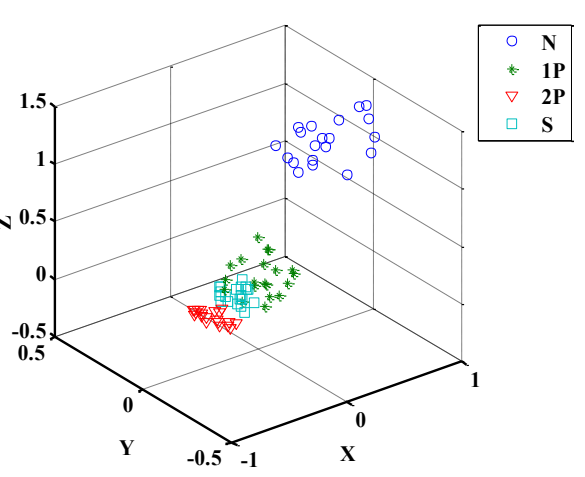

(b)

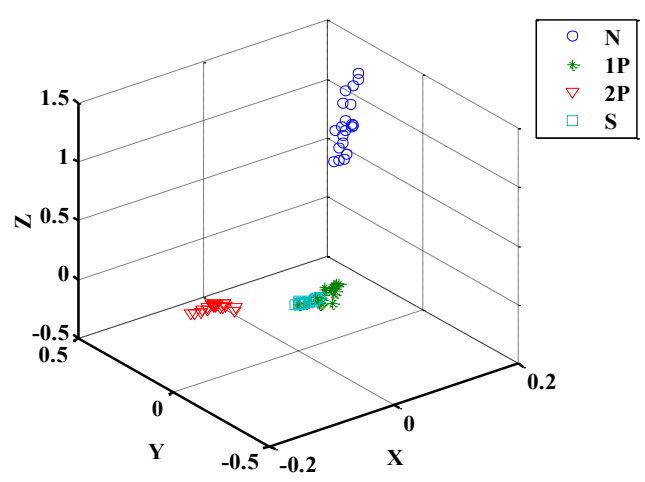

(c)

Fig. 9. (a) Features classification effect map of PCA - (b) Features classification effect map of LTSA - (c) Features classification effect map of LLTSA 


\subsection{Recognition of Fault Signals}

In order to verify the advantage of using fuzzy entropy of wavelet packet and LLTSA dimension reduction method in fault pattern recognition. Support vector machine (SVM) method was used for classification recognition to the approximate entropy of wavelet packet, fuzzy entropy of wavelet packet and wavelet packet fuzzy entropy eigenvalue after dimensionality reduction by the PCA, LTSA, LLTSA methods. In the process of the SVM pattern recognition, 20 sets of data in each case was selected as the training samples, and 20 sets as the test samples; "One-to-one" multiple classification algorithm was used in tests, radial basis function (RBF) was selected as kernel function, penalty parameter $\mathrm{c}$ and nuclear parameter $\mathrm{g}$ were the best parameters which obtained from particle swarm optimization; The training labels of normal hydraulic pump, wear-out failure of single loose slipper, double loose slipper and piston shoes were set to $1,2,3$, and 4 . Table 2 is about the recognition results by SVM with the various methods, A represents testing samples, $\mathrm{R}$ represents samples which had been identified correctly.

Table 2. Support vector machine recognition results

\begin{tabular}{|l|c|c|c|c|c|c|c|c|c|}
\hline \multicolumn{1}{|c|}{ Condition } & \multicolumn{2}{c|}{$\mathbf{N}$} & \multicolumn{2}{c|}{$\mathbf{1 P}$} & \multicolumn{2}{c|}{ 2P } & \multicolumn{2}{c|}{ S } & rate(\%) \\
\hline & $\boldsymbol{A}$ & $\boldsymbol{R}$ & $\boldsymbol{A}$ & $\boldsymbol{R}$ & $\boldsymbol{A}$ & $\boldsymbol{R}$ & $\boldsymbol{A}$ & $\boldsymbol{R}$ & \\
\hline AppEn & 20 & 18 & 20 & 11 & 20 & 19 & 20 & 16 & $78.75 \%$ \\
\hline SampEn & 20 & 17 & 20 & 12 & 20 & 20 & 20 & 19 & $85 \%$ \\
\hline FuzzyEn & 20 & 18 & 20 & 16 & 20 & 20 & 20 & 18 & $90 \%$ \\
\hline PCA-FuzzEn & 20 & 20 & 20 & 17 & 20 & 20 & 20 & 16 & $91.25 \%$ \\
\hline LTSA-FuzzEn & 20 & 20 & 20 & 17 & 20 & 20 & 20 & 17 & $92.5 \%$ \\
\hline LLTSA-FuzzEn & 20 & 20 & 20 & 19 & 20 & 20 & 20 & 18 & $96.25 \%$ \\
\hline
\end{tabular}

The analysis of SVM identification results in table 2 shows the following results: firstly, recognition rate of fault feature which used the fuzzy entropy of wavelet packet is much higher than approximate entropy and sample entropy, which is consistent with the analysis of fig. 5. It shows that the fuzzy entropy has a larger advantage in description of sample fault characteristics; Secondly, recognition rates of wavelet packet fuzzy entropy fault eigenvectors are higher after dimensionality reduction by the PCA, LTSA, LLTSA methods and increased by $1.25 \%, 2.5 \%$ and $6.25 \%$; Finally, the LLTSA method has higher identification accuracy than PCA and LTSA which testifies that as a kind of popular nonlinear learning method, the LLTSA keeps lowdimensional main characteristics of high-dimensional fault information in the process of dimensionality reduction, and can effectively distinguish between four kinds of fault state of hydraulic pump. Above analysis results show that integrated with wavelet packet decomposition, fuzzy entropy and LLTSA data dimension reduction method, multiple failure state of hydraulic pump can better recognized.

\section{Conclusion}

In this paper, a fault diagnosis model is presented to realize fault diagnosis of hydraulic pump system, this approach combined wavelet packet analysis techniques, 
fuzzy entropy, LLTSA (liner local tangent space alignment) and SVM. Wavelet packet analysis can provide a more sophisticated method for signal analysis. Fuzzy entropy is an improved version of sample entropy and has a better statistical stability. LLTSA is a new kind of manifold learning algorithm. Compared to PCA, LTSA algorithm, it has a better dimension reduction ability. Based on the multi-resolution characteristics of wavelet packet analysis, the statistical stability of fuzzy entropy and LLTSA's ability to extract the main features of nonlinear low-dimensional features, these methods are applied to the fault diagnosis of hydraulic pumps. Experimental results show that the proposed method is superior to wavelet packet approximate entropy, wavelet packet entropy, PCA fuzzy entropy and LTSA fuzzy entropy method. The fault diagnosis model can realize the pattern recognition of failure states of the hydraulic pump.

\section{$5 \quad$ References}

[1] Z.J He, J.Chen, T.Y Wang(1999).Theories and Applications of Machinery Fault Diagnostics, Science Press, China.

[2] Y. Guan, Y. Guo, X. Shui, Y.F Zhang(2014). Energy Analysis of Ertan Dam Seismic Response Based on Wavelet Packet Transform, Journal of Vibration, Measurement\& Diagnosis, $34: 1159-1163$.

[3] W. Zhao, L. Zhang, X.Meng (2011). Rolling bearing fault diagnosis using neural networks based on wavelet packet characteristic entropy, Communications in Computer and Information Science, $32: 424-429$ https://doi.org/10.1007/978-3-642-22691-5 74

[4] J.Qu, Z.Zhang, Z.He(2013). Mechanical Fault Diagnosis Model Based on Multi-wavelet Packet and Neighborhood Rough Set. Journal of Vibration ,Measurement \&Diagnosis, 33: 137-140.

[5] Ekici S, Yildirim S, Poyraz M(2008). Energy and entropy-based feature extraction for locating fault on transmission lines by using neural network and wavelet packet decomposition,Expert Systems with Applications, 34 : 2937-2944. https://doi.org/10.1016/j.eswa.200 7.05.011

[6] R.Yan, R.Gao(2007) Approximate entropy as a diagnostic tool for machine health monitoring. Mech. Syst. Signal Processing, 21: $824-839$. https://doi.org/10.1016/j.ymssp.2006 .02 .009

[7] Y.Yang, H.Pan, J Cheng(2013). The Application of VPMCD and Fuzzy Entropy in Rotor System Fault Diagnosis,Journal of Vibration, Measurement \& Diagnosis, 33: 137-140.

[8] Richman J S, Moorman J R(2000). Physiological time-series using approximate and sample entropy,Heart and Circulatory Physiology, 278: 2039-2019. https://doi.org/10.1152/ajpheart.2000.278.6.H2039

[9] W.Yang, P.Zhang (2015). Gear fault diagnosis based on multiscale fuzzy entropy of EEMD, Journal of Vibration and Shock, 34: 91- 94

[10] J.Zheng, M.Chen, J Cheng, Y.Yang(2014). Multiscale fuzzy entropy and its application in rolling bearing fault diagnosis, Journal of Vibration Engineering, 27: 145-149.

[11] Silva V D , Tenenbaum J B(2002) Global versus local methods in no-linear dimensionality reduction, Advances in Neural Information Processing Systems,51(3) 705-712

[12] D.Xiang, S.Ge(2014). Method of fault feature extraction based on EMD sample entropy and LLTSA, Journal of Aerospace Power, 29(7) 1535-1542. 
Paper-Fault Diagnosis Method for Hydraulic Pump Based on Fuzzy Entropy of Wavelet Packet and ...

[13] D.Xiang, S.Ge(2014). A model of fault feature extr(7) action based on wavelet packet sample entropy and manifold learning. Journal of Vibration and Shock, 33:1-5.

[14] X Richman J S , Moorman J R(2000) Physiological time-series analysis using approximate entropy and sample entropy, American Journal of Physiology Heart and Circulatory Physiology. 278: 2039 -2049. https://doi.org/10.1152/ajpheart.2000.278.6.H2039

[15] X Kosmidou V E, Hadjileontiadis L(2010). Using sample entropy for automated sign language recognition on s EMG and accelerometer data, Medical .Biological. Eng. Computing, 48: $255-267$.

[16] L.Zhang,P. Li, M.Li. Fault diagnosis of rolling bearing based on IDT fuzzy entropy and GG clustering, Chinese Journal of Scientific Instrument, 35(11)(2014) 2624-2632.

[17] Pincus S.M(2002). Assessing serial irregularity and its implications for health[J]. Acad.Sci: 954-245.

[18] W. Chen, Z.Wang, H.Xie (2007). Characterization of surface EMG signal based on fuzzy entropy.IEEE Transaction on Neural Systems and Rehabilitation Engineering. 15: 267272.

[19] Z.Zhao, S.YANG (2012). Sample entropy-based roller bearing fault diagnosis method. Journal of Vibration and Shock, 31: 136-140.

\section{Authors}

Wang Fei is a PhD student in Mechanical Engineering College, Shijiazhuang, China. His main research direction is mechanical testing technology. (e-mail: m15536259885@163.com).

Fang Liqing is a professor in Mechanical Engineering College, Shijiazhuang, China. His main research direction is the mechanical performance testing and fault diagnosis technology.

Qi Ziyuan is an associate professor in Mechanical Engineering College, Shijiazhuang, China. His main research direction is mechanical fault prediction technology.

Article submitted 16 October 2017. Resubmitted 15 November 2017. Final acceptance 04 February 2018. Final version published as submitted by the authors. 